\title{
Activation of cytoplasmic trehalase by cyclic- AMP-dependent and cyclic-AMP-independent signalling pathways in the yeast Candida utilis
}

\author{
D. Carrillo, J. Vicente-Soler, J. Fernandez, T. Soto, J. Cansado \\ and M. Gacto
}

Author for correspondence: M. Gacto. Tel: +3468 307100. Fax: +3468363963.

Department of Genetics and Microbiology, Facultad de Biologla, University of Murcia, 30071 Murcia, Spain

\begin{abstract}
Derepressed cells of Candida utilis suspended in buffer exhibited both a transient CAMP-mediated signal and a marked activation of cytoplasmic trehalase when supplemented with glucose. Nitrogen sources or protein synthesis inhibitors, as well as protonophores or uncouplers, were also able to cause trehalase stimulation in derepressed cells even in the absence of the sugar. The increase in trehalase activity caused by nitrogen sources or protein synthesis inhibitors was not accompanied by changes in CAMP levels. Moreover, acridine orange inhibited both the CAMP signal and the glucoseinduced activation of trehalase without affecting the increase in trehalase activity caused by nitrogen sources or protein synthesis inhibitors. These results suggest that CAMP is not involved as second messenger in the signal for trehalase stimulation induced by the latter compounds. By contrast, the addition of glucose to repressed cells suspended in buffer failed to cause the CAMP-mediated glucose signal and sugar-induced trehalase activation. No significant changes in either trehalase activity or CAMP concentration were observed upon addition to these cells of asparagine, cycloheximide, anisomycin or other agents, including protonophores and uncouplers. However, heat treatment of repressed cultures resulted in a moderate increase in trehalase activity with negligible change in CAMP levels, whereas such an effect was not observed in derepressed cultures. The thermally induced increase in trehalase activity was dependent on de novo protein synthesis and required the presence of glucose. Since in all cases the enzyme activated in vivo was deactivated in vitro by phosphatase our data support the idea that in C. utilis there are at least three independent mechanisms to increase trehalase activity, involving different, but overlapping, phosphorylation pathways.
\end{abstract}

Keywords: trehalase, glucose signal, cyclic AMP, Candida utilis, enzyme activation

\section{INTRODUCTION}

In many eukaryotic systems cyclic AMP (cAMP) plays a central role in signal transduction in response to several extracellular stimuli. In the budding yeast Saccharomyces cerevisiae one of the best characterized signalling pathways, although not entirely understood, is the glucose-induced cAMP-mediated pathway that controls the activity of various key enzymes involved in carbon metabolism by cAMP-dependent protein phosphorylation (reviewed by Wills, 1990; Thevelein, 1991, 1992). Activation of the cAMP pathway in Sacch. cerevisiae leads to many effects, apparently unrelated, including a shift from gluconeogenic to glycolytic metabolism, failure to arrest in the G1 phase of the cell cycle, low levels of storage carbohydrates or sensitivity to heat shock treatment (Kataoka et al., 1984; Cameron et al., 1988). The cytoplasmic enzyme trehalase (EC 3.2.1.28) is also under regulatory control by the glucose signal transduction pathway (reviewed by Thevelein, 1988). Phosphorylation of trehalase causes enzyme activation and changes in the pool of trehalose accumulated by yeast cells upon nutrient starvation or physical stress. The trehalose content determines cell survival under a variety of environmental conditions (reviewed by Van Laere, 1989; Wiemken, 1990).

Although the glucose-triggered signal for trehalose mobilization has been extensively studied in Sacch. cere- 
visiae our knowledge of the physiological significance of this response is almost exclusively restricted to this species and practically nothing is known in distantly related yeasts. The general occurrence of this transduction pathway among yeasts needs to be assessed using a wider range of species. Recently, we have found an equivalent cAMP-mediated glucose-induced signal in the fission yeast Schizosaccharomyces pombe (Carrillo et al., 1994). In this context, previous studies have shown that the neutral trehalase in derepressed cells of Candida utilis can be activated by glucose and that this activation correlates with phosphorylation of the enzyme protein (Arguelles \& Gacto, 1985; Arguelles et al., 1986). Such data suggested the existence in this yeast of a cAMP signalling pathway induced by glucose and working by activation of cAMPdependent protein kinase, similar to that described in Sacch. cerevisiae (Thevelein, 1991, 1992). In this work we present additional evidence supporting the existence in $C$. utilis of a cAMP signalling pathway analogous, although not identical, to that present in Sacch. cerevisiae, one of whose final molecular targets is also the cytoplasmic trehalase. Moreover, we have analysed the role of cAMP in the stimulation of trehalase by other agents such as nitrogen sources, protein synthesis inhibitors or heat shock to reveal the occurrence in this yeast of alternative pathways for increased trehalase activity that are independent of drastic changes in the concentration of cAMP.

\section{METHODS}

Organism and growth conditions. Candida utilis ATCC 60459 was grown at $30^{\circ} \mathrm{C}$ on a rotary shaker operated at 160 r.p.m. in medium containing $1 \%(\mathrm{w} / \mathrm{v})$ yeast extract, $2 \%(\mathrm{w} / \mathrm{v})$ peptone and either $2 \%(\mathrm{w} / \mathrm{v})$ glucose (YPD medium) or $3 \%(\mathrm{v} / \mathrm{v})$ glycerol (YPG medium). Repressed cells were harvested from cultures growing exponentially in YPD $\left(\mathrm{OD}_{600}=1 \cdot 0-1 \cdot 5\right)$. Derepressed cells were collected from stationary-phase cultures in YPD after glucose exhaustion $\left(\mathrm{OD}_{600}=12.0\right)$ or from cultures growing exponentially in YPG $\left(\mathrm{OD}_{600}=3 \cdot 0-4 \cdot 5\right)$. The cells were washed with cold distilled water and resuspended in $100 \mathrm{mM}$ sodium acetate buffer, $\mathrm{pH} 5 \cdot 6$, at a concentration of $25 \mathrm{mg}$ (wet wt) $\mathrm{ml}^{-1}$ (approximately $7 \times 10^{8}$ cells $\mathrm{ml}^{-1}$ ).

Induction of enzyme activation and preparation of cell-free extracts. In time-course experiments, suspensions of repressed or derepressed cells were incubated in a shaking water bath and allowed to equilibrate at $30^{\circ} \mathrm{C}$ for $5 \mathrm{~min}$. Before and after addition of the compounds assessed for triggering trehalase activation (zero time), aliquots were removed at timed intervals and the cells washed as above in the cold. Cell-free extracts were prepared and centrifuged as previously described (Carrillo $e t$ al.,

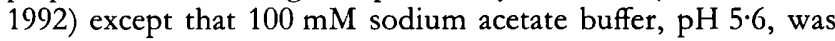
used to avoid the simultaneous measurement of both vacuolar and cytoplasmic trehalases in assays containing this buffer. Acetate specifically inhibits the vacuolar trehalase present in this organism (Arguelles \& Gacto, 1985, 1988). For trehalase activation by heat shock treatment, either repressed or derepressed cultures were shifted at $40^{\circ} \mathrm{C}$ following the procedure of De Virgilio et al. (1990).

Trehalase assays and measurement of cAMP. Trehalase activity was determined as previously described (Arguelles \& Gacto, 1985). One unit of trehalase activity catalysed the release of $1 \mathrm{nmol}$ glucose $\mathrm{min}^{-1}$ under the assay conditions. Protein was determined by the Lowry method. When acridine orange was used, because the residual levels of this drug interfere severely with the protein determination method, the protein contents measured for the controls were also used for the drugtreated samples. cAMP was extracted from the cells as described by Arkinstall et al. (1991) and the cAMP content determined using the Amersham $\left[{ }^{3} \mathrm{H}\right]$ cAMP radioassay kit as reported elsewhere (Carrillo et al., 1994). Trehalase and cAMP levels were always determined in parallel on the same cell sample.

Deactivation of trehalase in vitro by phosphatase. After activation in vivo by various compounds, deactivation of trehalase was accomplished by treatment at $37^{\circ} \mathrm{C}$ with proteasefree alkaline phosphatase as described previously (Arguelles \& Gacto, 1985).

Reproducibility of results. All experiments were repeated at least three times with similar results. Representative results are shown.

\section{RESULTS}

\section{Trehalase and CAMP in derepressed cells}

The addition of glucose to derepressed cells of C. utilis suspended in buffer triggered a marked activation of trehalase (about fivefold) which was preceded by a twofold increase in the concentration of cAMP (Figs 1 and 2). No significant increase in trehalase activity was detected during the first $10 \mathrm{~min}$ of incubation. In the absence of sugar, derepressed cells of this yeast also showed activation of trehalase upon addition of 2,4dinitrophenol (DNP), iodoacetate, sodium azide, asparagine, cycloheximide or anisomycin (Figs 1,2 and 3). The stimulatory effect of some of these compounds on trehalase was even higher than that induced by glucose.

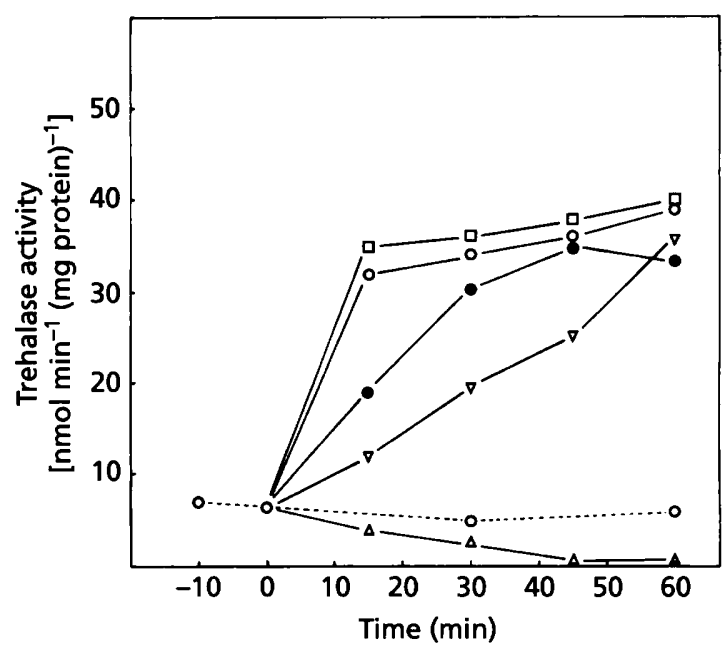

Fig. 1. Activation of trehalase in derepressed cells of $C$. utilis by addition of $100 \mathrm{mM}$ glucose $(0), 0.19 \mathrm{mM}$ anisomycin $(\nabla)$, $2 \mathrm{mM}$ DNP $(O)$ or $10 \mathrm{mM}$ iodoacetate $(\square)$. Trehalase in control cells from suspensions without additions is represented by a discontinuous line. As a negative effector, $2 \mathrm{mM}$ copper sulphate $(\triangle)$ was added. 


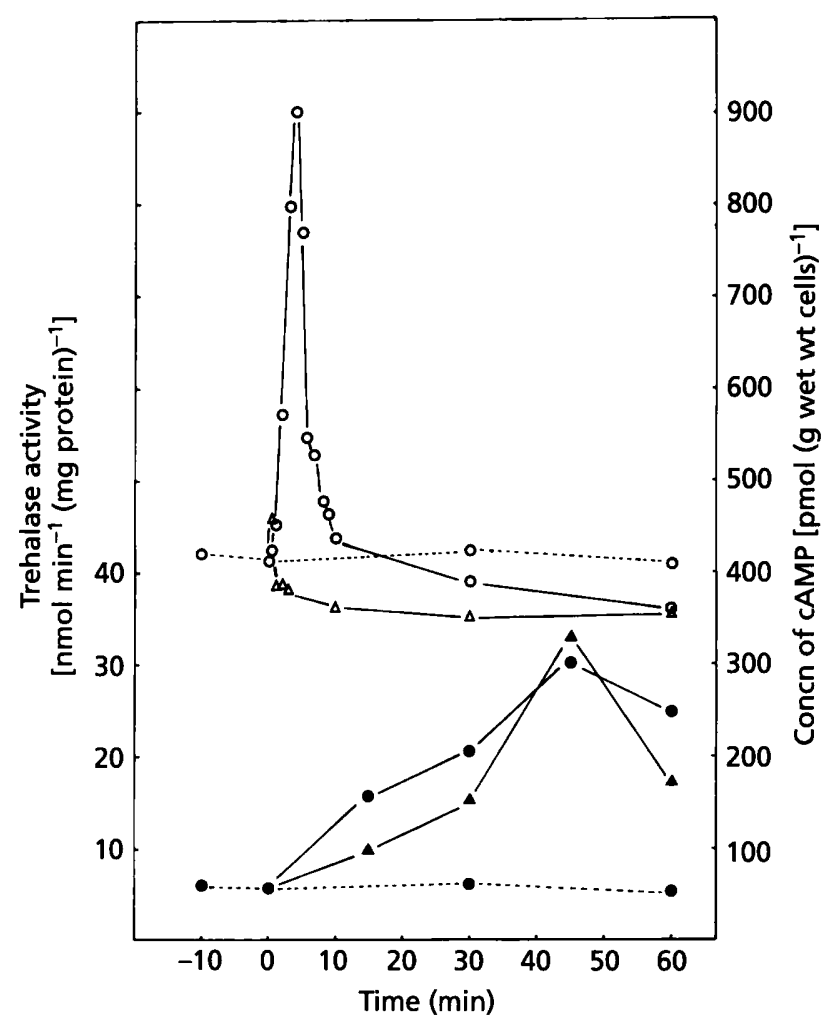

Fig. 2. Changes in trehalase activity (filled symbols) and CAMP levels (open symbols) upon addition to derepressed cells of $100 \mathrm{mM}$ glucose $(0,0)$ or $10 \mathrm{mM}$ asparagine $(\Delta, \Delta)$. Trehalase activity and CAMP levels in control cells from suspensions not supplemented with carbon or nitrogen sources are represented by discontinuous lines.

Trehalase activation by sodium azide (Fig. 3), iodoacetate or DNP (not shown) was accompanied by a clear cAMP signal. This fact might reflect a higher ATP content in these cells than in derepressed cells of Schiz. pombe, where trehalase activation by the same agents is energy-dependent and requires an exogenous supply of sugar (Carrillo et al., 1992).

In Sacch. cerevisiae asparagine, cycloheximide or anisomycin are able to cause activation of trehalase, but only in the presence of glucose (Thevelein \& Beullens, 1985; Hirimburegama et al., 1992). In derepressed cells of $C$. utilis, the aforementioned compounds were also able to trigger activation of trehalase (Figs 1,2 and 3) and, as in Sacch. cerevisiae (Thevelein \& Beullens, 1985), the activation signal produced by either the nitrogen source or the protein synthesis inhibitors did not involve changes in the level of intracellular cAMP (Figs 2 and 3). However, the mechanism underlying this stimulation does not appear to be the same in the two yeasts. In C. utilis we found that, unlike Sacch. cerevisiae the activation does not require the presence of glucose and is only clearly observed in derepressed cells (see below).

To verify further that the process of trehalase activation by nitrogen sources or protein synthesis inhibitors takes

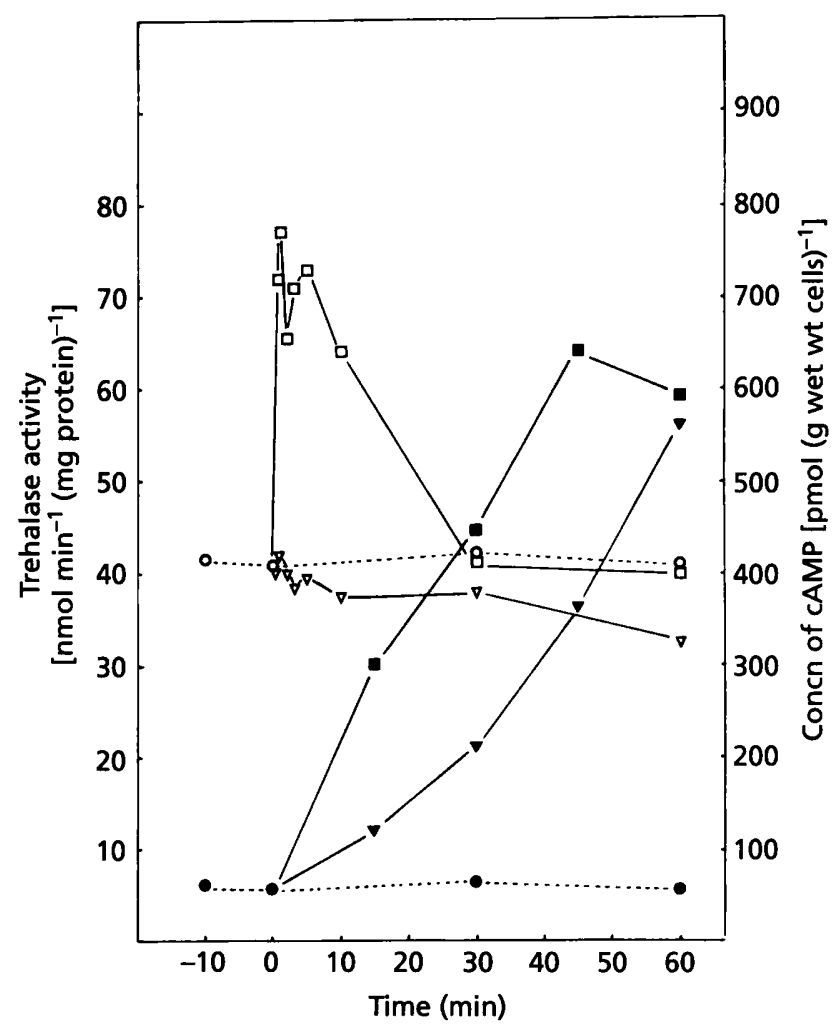

Fig. 3. Changes in trehalase activity (filled symbols) and CAMP levels (open symbols) upon addition to derepressed cells of $2 \mathrm{mM}$ sodium azide $(\square, \square)$ or $0.18 \mathrm{mM}$ cycloheximide $(\nabla, \nabla)$. Trehalase activity and CAMP levels in control cells are represented by discontinuous lines.

place without a previous transient cAMP signal we repeated the above experiments in the presence of acridine orange. This drug has been shown to suppress the cAMP signal in Sacch. cerevisiae (François et al., 1984; Thevelein \& Beullens, 1985). In C. utilis, acridine orange inhibited both the increase in the cAMP level and the increase in the activity of trehalase induced by glucose, thus confirming the role of the cAMP signal as second messenger in the sugar-induced activation of trehalase (Fig. 4). However, this drug was unable to inhibit the activation of trehalase induced by asparagine (Fig. 5). Similarly, the presence of acridine orange had no significant effect on the increase induced by cycloheximide or anisomycin (results not shown).

\section{Trehalase and CAMP in repressed cells}

Confirming previous results (Arguelles \& Gacto, 1985), cells of $C$. utilis harvested from cultures growing exponentially on glucose did not increase trehalase activity in response to the addition of the sugar. As shown in Fig. 6 , these cells also lacked the ability to activate trehalase in the presence of asparagine, sodium azide, DNP, iodoacetate or protein synthesis inhibitors. Instead of increases, slow decreases in trehalase activity were often 


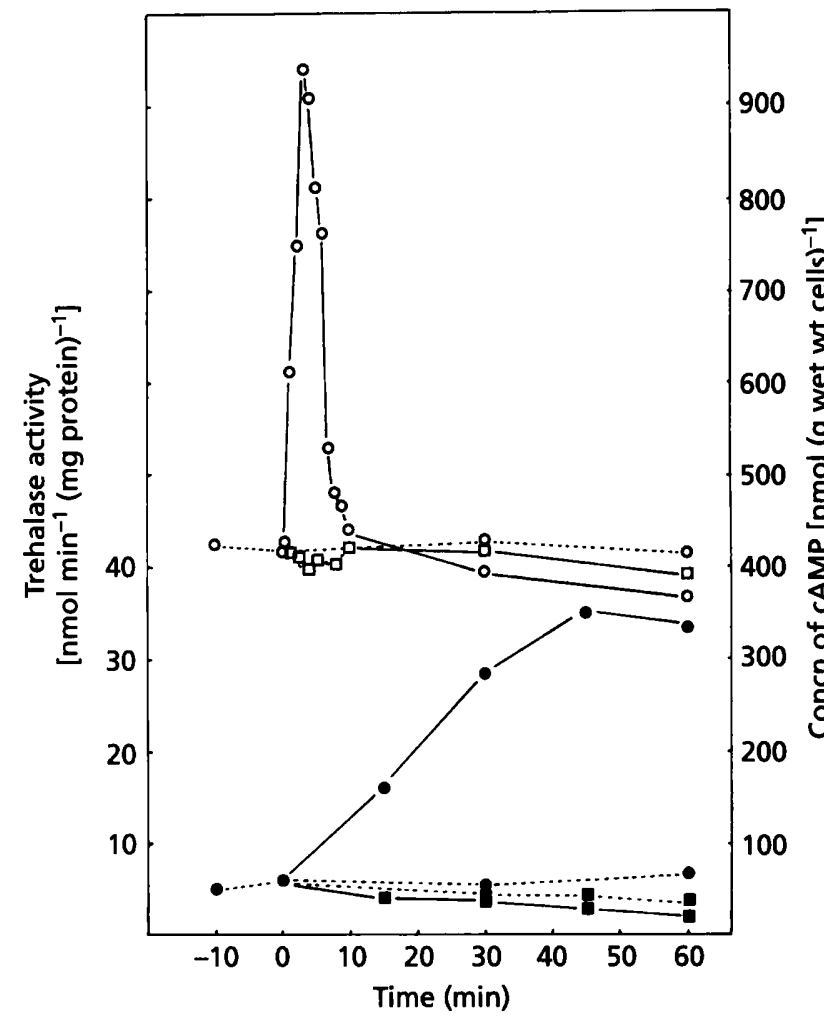

Fig. 4. Effect of acridine orange on glucose-induced trehalase activation and the CAMP signal. At zero time $100 \mathrm{mM}$ glucose was added to a suspension of derepressed cells in incubation buffer in the absence $(0,0)$ or presence $(\square, \square)$ of $2 \mathrm{mM}$ acridine orange added $10 \mathrm{~min}$ before glucose. Trehalase activity (filled symbols) and cAMP levels (open symbols) were measured at timed intervals. Results from control cell suspensions maintained without adding dye and glucose or supplemented only with the dye are represented by discontinuous lines.

recorded when each of these compounds was added as a potential inducer of trehalase activation and this effect was particularly marked following the addition of the protonophore DNP (Fig. 6). None of these additions was accompanied by increases in cAMP content above the basal level, which was in the range of $700-800 \mathrm{pmol}(\mathrm{g}$ wet wt cells) ${ }^{-1}$. Since it could be argued that repressed cells of $C$. utilis suspended in buffer are devoid of sufficient energy charge to produce a cAMP signal in response to the above compounds, we performed the same type of experiments by adding glucose to the suspension buffer $10 \mathrm{~min}$ in advance of the different inducers. Again, no significant increases in either trehalase activity or cAMP content were recorded in cells suspended in glucose-containing buffer (results not shown).

\section{Effect of heat shock on trehalase and CAMP}

Heat shock treatment of repressed cultures resulted in a moderate (about twofold) but reproducible increase in trehalase activity (Fig. 7). This change in enzyme activity was not associated with any significant increase in the cAMP level (Fig. 7). Heat treatment of cell extracts did

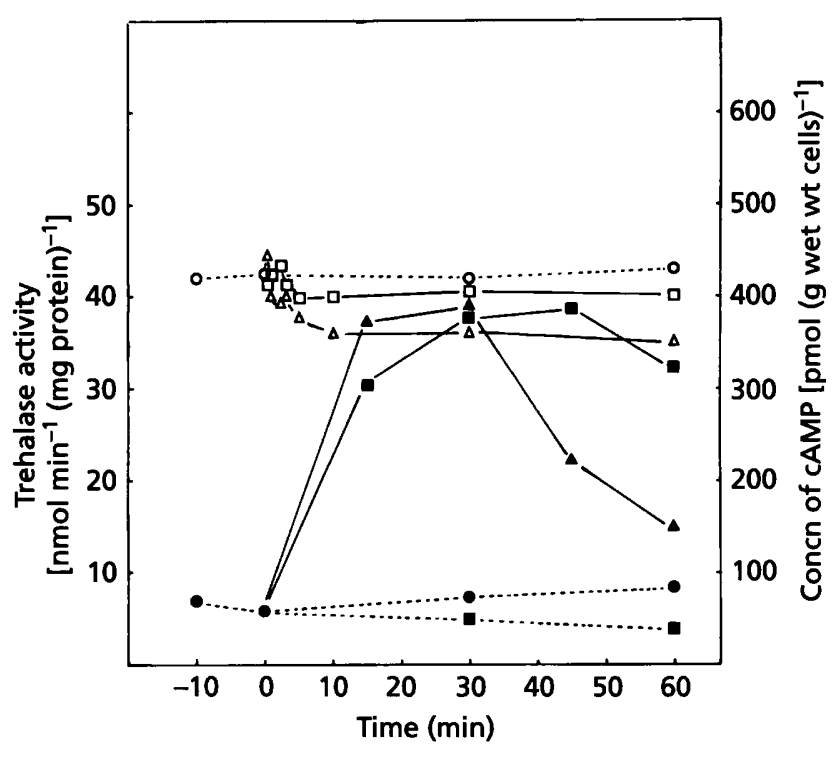

Fig. 5. Effect of acridine orange on asparagine-induced trehalase activation and corresponding CAMP levels. At zero time $10 \mathrm{mM}$ asparagine was added to a suspension of derepressed cells in incubation buffer in the absence $(\Delta, \Delta)$ or presence $(\square, \square)$ of $2 \mathrm{mM}$ acridine orange added $10 \mathrm{~min}$ before asparagine. Trehalase activity (filled symbols) and CAMP levels (open symbols) were measured at timed intervals. Results in control cells from suspensions not supplemented with the dye and asparagine or supplemented only with the dye are represented by discontinuous lines.

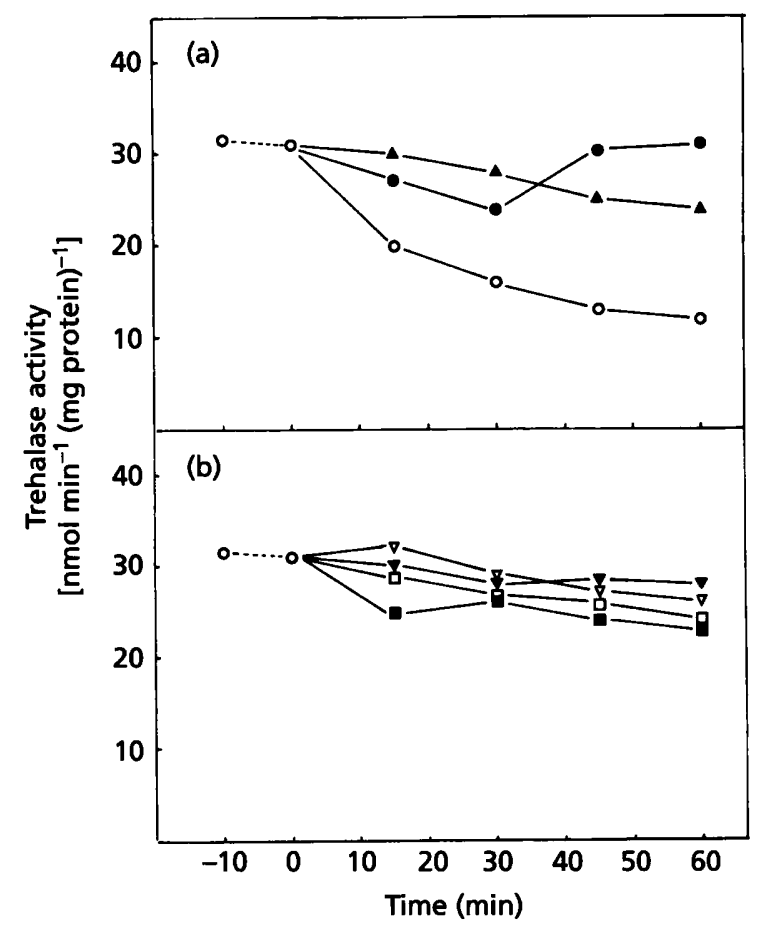

Fig. 6. Effect of the addition of various compounds on trehalase activity in repressed cells. (a) $0,100 \mathrm{mM}$ glucose; $\Delta$, $10 \mathrm{mM}$ asparagine; $O, 2 \mathrm{mM}$ DNP. (b) $\nabla, 0.18 \mathrm{mM}$ cycloheximide; $\nabla, 0.19 \mathrm{mM}$ anisomycin; $\square, 10 \mathrm{mM}$ iodoacetate; 口, $2 \mathrm{mM}$ sodium azide. 


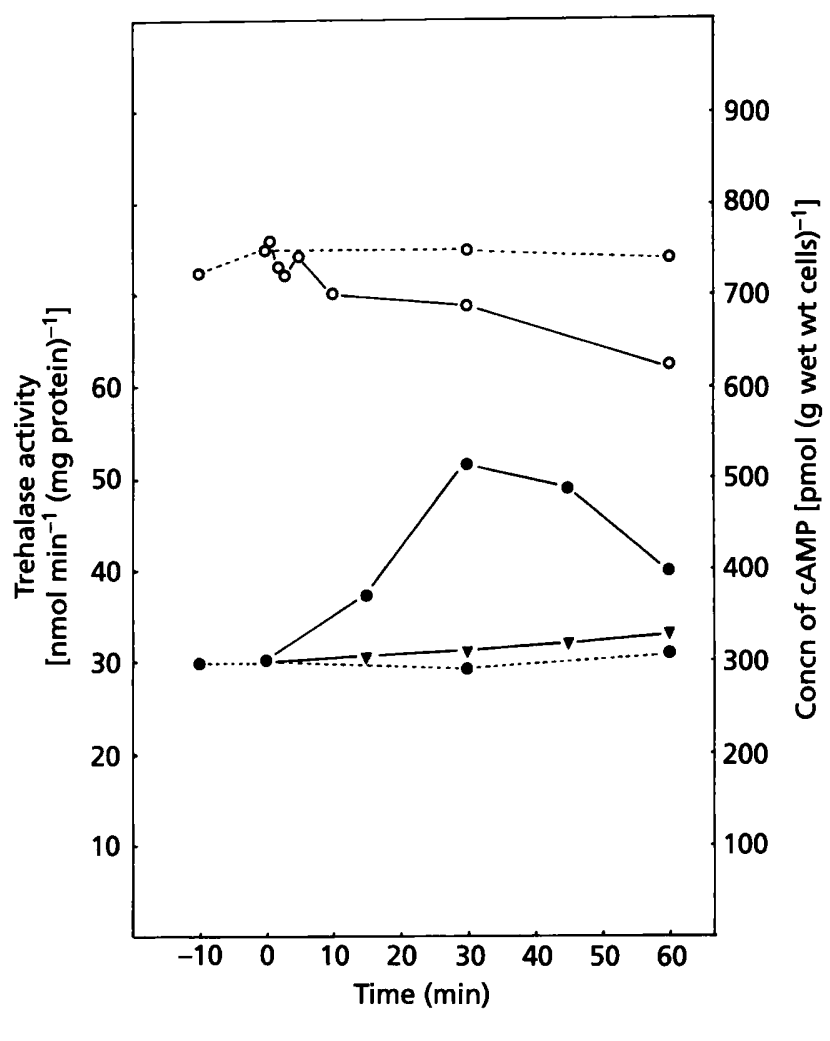

Fig. 7. Trehalase activity (filled symbols) and corresponding CAMP levels (open symbols) in repressed cells during a heat shock. Exponential-phase cultures growing on glucose were either shifted at zero time to $40^{\circ} \mathrm{C}$ in the absence $(0,0)$ or presence $(\nabla)$ of $0.18 \mathrm{mM}$ cycloheximide or maintained at $28{ }^{\circ} \mathrm{C}$ as a control. Trehalase activity and CAMP levels in control cells are represented by discontinuous lines.

not result in activation of trehalase in vitro suggesting that the phenomenon was not a direct thermal effect on the enzyme protein.

As described in other yeasts (De Virgilio et al., 1990), the increase in trehalase activity induced by heat shock might be due to regulation at a post-translational level. To test this hypothesis, we added $0.35 \mathrm{mM}$ cycloheximide to repressed growing cultures $10 \mathrm{~min}$ before starting the thermal treatment. Control experiments performed in parallel confirmed the effective inhibition of protein synthesis and the arrest in growth of the cultures under the heat shock conditions. Interestingly, no increase in trehalase activity by heat shock was obtained in the presence of cycloheximide (Fig. 7) indicating that the effect induced by heat treatment might be due to either newly formed trehalase or modification of pre-existing enzyme by some factor synthesized de novo during the activation period. The presence of glucose in the medium was required for successful activation during the heat shock. Acetate or glycerol, which sustain rapid growth of this yeast, were nevertheless unable to substitute for the sugar as an alternative carbon source.

Although the response of repressed cultures to heat shock

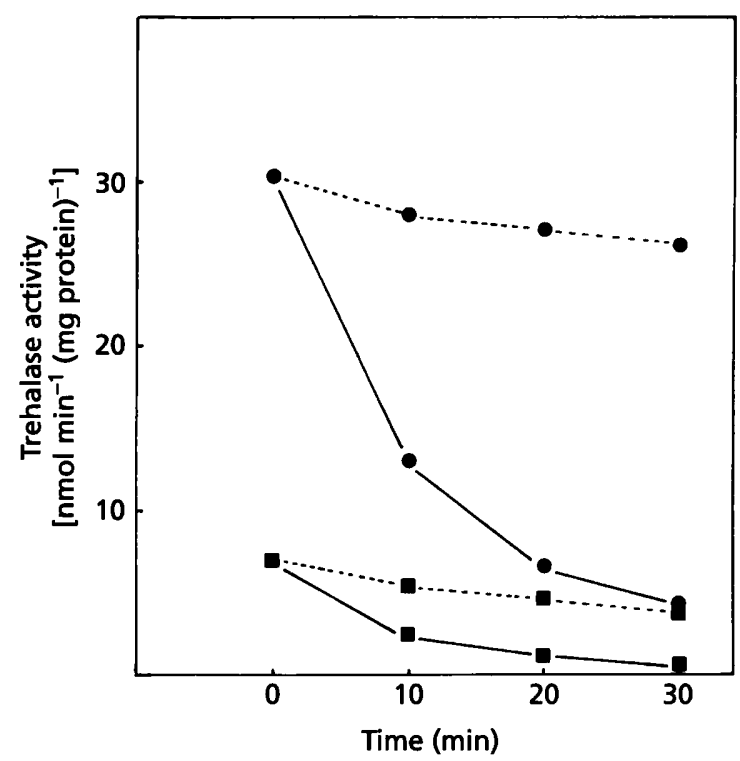

Fig. 8. Deactivation of trehalase by phosphatase. Cell extracts from repressed $(\Theta)$ or derepressed cells $(\square)$ were treated with phosphatase $(\longrightarrow)$ or buffer alone $(---)$ as indicated in Methods.

was clear cut (Fig. 7) no significant increase in trehalase activity was produced when the thermal treatment was applied to derepressed cultures (results not shown). This is in contrast to Sacch. cerevisiae, where activation of trehalase by heat shock has been reported in both repressed and derepressed cells (Hottiger et al., 1987; Panek et al., 1989).

\section{Deactivation by phosphatase of trehalase activated in vivo}

We have previously demonstrated the activation and simultaneous phosphorylation of trehalase in C. utilis by addition of glucose (Arguelles et al., 1986). Cell-free extracts of this yeast exhibit a slow, time-dependent decrease in trehalase presumably due to cellular phosphatases and/or proteinases. The rate of loss in trehalase activity is greatly increased by exogenous phosphatase (Fig. 8). This result strongly supports the idea that dephosphorylation of the enzyme results in loss of activity. As shown in Table 1, trehalase activated in vivo by any of the various procedures mentioned above was deactivated in vitro by alkaline phosphatase, indicating that phosphorylation of the enzyme protein may be a general phenomenon associated with trehalase activation by different stimuli. Since the transduction signal for trehalase activation induced by nitrogen sources, protein synthesis inhibitors or heat shock does not appear to include a cAMP signal (Figs 2, 3 and 7) the possibility of cAMP-independent phosphorylation has to be considered in these cases. 
Table 1. Deactivation of trehalase by phosphatase

Alkaline phosphatase (12 $\mathrm{U}$ ) was added to $0.4 \mathrm{ml}$ cell extract from derepressed cells containing $1 \mathrm{mg}$ protein and incubated for $15 \mathrm{~min}$. Aliquots were then removed and the remaining activity determined in standard trehalase assays. Except for the heat shock treatment, derepressed cells were used.

\begin{tabular}{|c|c|c|c|}
\hline \multirow[t]{2}{*}{ Activating agent } & \multicolumn{2}{|c|}{$\begin{array}{c}\text { Trehalase activity } \\
\text { [units (mg protein) }^{-1} \text { ] }\end{array}$} & \multirow[t]{2}{*}{$\begin{array}{c}\text { Deactivation } \\
(\%)\end{array}$} \\
\hline & Control & + Phosphatase & \\
\hline Glucose $(0 \cdot 1 \mathrm{M})$ & $27 \cdot 8$ & $10 \cdot 1$ & $63 \cdot 7$ \\
\hline Cycloheximide $(0 \cdot 18 \mathrm{mM})$ & $45 \cdot 3$ & $7 \cdot 3$ & $83 \cdot 9$ \\
\hline Iodoacetic acid $(10 \mathrm{mM})$ & $32 \cdot 1$ & $4 \cdot 9$ & $84 \cdot 8$ \\
\hline Sodium azide ( $2 \mathrm{mM})$ & $53 \cdot 0$ & $17 \cdot 5$ & $67 \cdot 0$ \\
\hline Asparagine $(10 \mathrm{mM})$ & $15 \cdot 6$ & $2 \cdot 0$ & $87 \cdot 2$ \\
\hline Dinitrophenol (2 mM) & $27 \cdot 7$ & $4 \cdot 2$ & $84 \cdot 9$ \\
\hline Anisomycin $(0 \cdot 19 \mathrm{mM})$ & $21 \cdot 7$ & $4 \cdot 0$ & $81 \cdot 6$ \\
\hline Heat shock $\left(40^{\circ} \mathrm{C}, 30 \mathrm{~min}\right)$ & $56 \cdot 6$ & $22 \cdot 3$ & $60 \cdot 7$ \\
\hline
\end{tabular}

\section{DISCUSSION}

Our results support the idea that at least three different mechanisms have evolved in $C$. utilis to increase trehalase activity in response to diverse nutritional or environmental conditions. One of them is only operative in repressed cells upon heat shock, requires glucose and protein synthesis and does not depend on changes in the intracellular concentration of cAMP. In C. utilis this heatinduced increase in trehalase activity may not correspond to activation of pre-existing enzyme but to enzyme formed de novo during the heat shock. On the other hand, the glucose-induced pathway requires the expression of some glucose-repressive component for activation of the target enzyme. Transduction of this signal appears to rely on a transient increase in cAMP and subsequent stimulation of cAMP-dependent protein kinase. This pathway can also be triggered by compounds other than sugars such as protonophores or uncouplers. Finally, the signal for activation produced by nitrogen sources or protein synthesis inhibitors is independent of drastic changes in cAMP concentration and mostly operative in derepressed cells. These results reveal both some similarities and some significant differences about what is known in other yeast species previously studied (Thevelein, 1991, 1992; Carrillo et al., 1994).

The existence in C. utilis of a glucose-induced cAMP signal transduction pathway similar to that characterized in Sacch. cerevisiae (Thevelein, 1991, 1992) was suspected previously (Arguelles \& Gacto, 1985; Arguelles et al., 1986). However, no direct measurement of cAMP levels immediately after glucose addition was performed in those studies to test whether the concentration of this nucleotide was in fact altered in response to glucose. Our present work indicates that glucose triggers a rapid and reversible change in the steady-state level of cAMP in derepressed cells, thus widening the significance of this response in yeasts. In $C$. utilis the steady-state level of cAMP in repressed cells is higher than in derepressed cells
(Figs 2 and 7) and, interestingly, the basal level of trehalase activity in repressed cells is also higher than in derepressed cells. This situation is different to that described in other yeasts (Carrillo et al., 1994). Whether the higher trehalase activity in repressed cells as compared to derepressed cells is due exclusively to a different degree of enzyme phosphorylation or to additional regulatory controls at transcriptional or post-translational levels is at present unknown.

With a few exceptions (Mansure et al., 1992), no studies have been published to compare the behaviour of trehalase, as a sensor for the cAMP signal, in Crabtreepositive and Crabtree-negative yeasts upon addition of glucose. In the nonfermenting yeast $R$ bodotorula rubra no response of trehalase to glucose was observed (Mansure $e t$ al., 1992). Therefore it seems noteworthy that in C.utilis, a Crabtree-negative yeast with a physiology so different from the strongly fermentative Sacch. cerevisiae (Van Urk et al., 1988), a similar pathway for trehalase activation by glucose is nevertheless present. The response of trehalase in this yeast appears to be slower than in Sacch. cerevisiae, where trehalase activation closely follows the cAMP peak (Thevelein \& Beullens, 1985). The stages between the initial interaction of glucose and the phosphorylation of trehalase in $C$. utilis remain unknown but the similarity of the response in the two yeasts may be extensive. However, several aspects concerning trehalase activation by agents other than glucose through this cAMP-dependent pathway appear to be markedly different in $C$. utilis. For instance, the protonophore DNP, which in both repressed and derepressed cells of Sacch. cerevisiae induces a cAMP signal and activation of trehalase (Arguelles et al., 1990), fails to enhance significantly the enzyme activity in repressed cells of $C$. utilis. A close examination of the effect of other compounds on repressed cells reveals that this is also true for sodium azide or iodoacetate as well as for nitrogen sources or protein synthesis inhibitors, even though these latter compounds use a different pathway for trehalase activation. It is unlikely that the failure to 
activate the enzyme in repressed cells by any of these agents might be due to low ATP content since the level of cAMP in these cells as compared to derepressed cells is not reduced. In addition, the presence of acetate in the suspension buffer should ensure a certain level of exogenous energy source for this yeast that is much less fermentative than Sacch. cerevisiae (Van Urk et al., 1988). Moreover, each of the compounds assayed was also unable to activate trehalase markedly when glucose was added to the suspension buffer in advance of the inducer agent. Rather, the apparent inability to induce significant activation in repressed cells might in part derive from the comparatively high steady-state level of cAMP already present in these cells and from the corresponding high levels of trehalase activity. In other words, most of the trehalase appears to be already activated in repressed cells and cannot be significantly increased any further. Also, the requirement for some glucose-repressible component in the mechanism of signal transduction, as it occurs in Sacch. cerevisiae and in Schiz. pombe (Thevelein, 1991, 1992; Carrillo et al., 1994), cannot be dismissed as an alternative explanation to account for the apparent insensitivity of repressed cells to these potential inducers.

Besides the cAMP-dependent pathway, other cAMPindependent signals causing activation of trehalase can also occur in $C$. utilis. The results indicating that protein synthesis inhibitors activate trehalase in this yeast without changes in cAMP are somewhat surprising. Cycloheximide causes variations in cAMP concentration in Schiz. pombe (Carrillo et al., 1994) but not in Sacch. cerevisiae (Thevelein \& Beullens, 1985). We proposed previously that the activation of trehalase in $C$. utilis after addition of glucose in the presence of cycloheximide was indicative of a modification of the enzyme as the biochemical event causing activation (Arguelles \& Gacto, 1985). However, because it is now clear that protein synthesis inhibitors are by themselves inducers of trehalase activation, the posttranslational nature of the stimulation triggered by compounds other than cycloheximide or anysomicin cannot be unequivocally established by such a criterion. Nevertheless, we favour the idea that the increase in enzyme activity caused by other inducers is also due to enzyme modification in view of the rather rapid kinetics of the activation response in cells maintained in buffer, the deactivation in vitro by phosphatase and the previous demonstration that, at least in the case of glucose, phosphorylation of the enzyme protein takes place during activation (Arguelles et al., 1986).

In Sacch. cerevisiae the activation of trehalase induced by nitrogen sources requires the presence of glucose (Thevelein \& Beullens, 1985). This nitrogen-induced trehalase activation is probably due to phosphorylation of the enzyme mediated by protein kinase without enhancing the cAMP level (Hirimburegama et al., 1992). Since in Sacch. cerevisiae the glucose-sensing system involved in glucose-induced activation of trehalase appears to be also required for the activation induced by nitrogen sources, the possibility that the same pathway could serve as a sensor for the presence of both types of nutrient has been suggested (Hirimburegama et al., 1992). However, our results clearly show that the nitrogen sources are able to trigger trehalase activation in $C$. utilis in the absence of sugar (Fig. 2). To our knowledge this is the first demonstration in yeasts of a response of trehalase induction by nitrogen sources that is independent of glucose. Such a difference is important because it casts some doubt on the general occurrence of a common sensing system. Also, in derepressed cells of Schiz. pombe (unpublished results), although not in the case of Sacch. cerevisiae (unpublished results; Thevelein \& Beullens, 1985) trehalase activation by nitrogen sources occurs in the absence of glucose provided that an alternative energy source, such as glycerol, is present. Hence, the association of glucose with a nitrogen source for stimulation by the latter of the pathway causing trehalase activation might not be a widespread requirement among yeasts.

\section{REFERENCES}

Arguelles, J. C. \& Gacto, M. (1985). Evidence for regulatory trehalase activity in Candida utilis. Can J Microbiol 31, 529-537.

Arguelles, J. C. \& Gacto, M. (1988). Differential location of regulatory and nonregulatory trehalases in Candida utilis cells. Antonie Leeunenboek 54, 555-565.

Arguelles, J. C., Vicente-Soler, J. \& Gacto, M. (1986). Protein phosphorylation and trehalase activation in Candida utilis. FEMS Microbiol Lett 34, 361-365.

Arguelles, J. C., Mbonyi, K., Van Aelst, L., Vanhalewyn, M., Jans, A. W. H. \& Thevelein, J. M. (1990). Absence of glucose-induced cAMP signaling in the Saccharomyces cerevisiae mutants cat 1 and cat 3 which are deficient in derepression of glucose-repressible proteins. Arch Microbiol 154, 199-205.

Arkinstall, S. J., Papasavvas, S. G. \& Payton, M. A. (1991). Yeast $\alpha$ mating factor receptor-linked G-protein signal transduction supresses Ras-dependent activity. FEBS Lett 284, 123-128.

Cameron, S., Levin, L., Zoller, M. \& Wigler, M. (1988). cAMPindependent control of sporulation, glycogen metabolism, and heat-shock resistance in S. cerevisiae. Cell 53, 555-566.

Carrillo, D., Vicente-Soler, J. \& Gacto, M. (1992). Activation of neutral trehalase by fermentable sugars and cAMP in the fission yeast Schizosaccharomyces pombe. FEMS Microbiol Lett 98, 61-66.

Carrillo, D., Vicente-Soler, J. \& Gacto, M. (1994). Cyclic AMP signalling pathway and trehalase activation in the fission yeast Schizosaccharomyces pombe. Microbiology 140, 1467-1472.

De Virgilio, C., Simmen, U., Hottiger, T., Boller, T. \& Wiemken, A. (1990). Heat shock induces enzymes of trehalose metabolism, trehalose accumulation, and thermotolerance in Schizosaccharomyces pombe, even in the presence of cycloheximide. FEBS Lett 273, 107-110.

François, J., Van Schaftingen, E. \& Hers, H. G. (1984). The mechanism by which glucose increases fructose-2,6-bisphosphate concentration in Saccharomyces cerevisiae. A cyclic-AMP-dependent activation of phosphofructokinase 2. Eur J Biochem 145, 187-193.

Hirimburegama, K., Durnez, P., Keleman, J., Oris, E., Vergauwen, R., Mergelsberg, H. \& Thevelein, J. M. (1992). Nutrient-induced activation of trehalase in nutrient-starved cells of the yeast Saccharomyces cerevisiae: cAMP is not involved as second messenger. $J$ Gen Microbiol 138, 2035-2043.

Hottiger, T., Schmutz, P. \& Wiemken, A. (1987). Heat-induced accumulation and futile cycling of trehalose in Saccharomyces cerevisiae. J Bacteriol 169, 5518-5522. 
Kataoka, T., Powers, S., McGill, C., Fasano, O., Strathern, J., Broach, J. \& Wigler, M. (1984). Genetic analysis of yeast $R A S 1$ and RAS2 genes. Cell 37, 437-445.

Mansure, J. J., Silva, J. T. \& Panek, A. D. (1992). Characterization of trehalase in Rhodotorula rubra. Biochem Int 28, 693-700.

Panek, A. D., Ferreira, R. \& Panek, A. C. (1989). Comparative studies between the glucose-induced phosphorylation signal and the heat shock response in mutants of Saccharomyces cerevisiae. Biocbimie 71, 313-318.

Thevelein, J. M. (1988). Regulation of trehalase activity by phosphorylation-dephosphorylation during developmental transitions in fungi. Exp Mycol 12, 1-12.

Thevelein, J. M. (1991). Fermentable sugars and intracellular acidification as specific activators of the RAS-adenylate cyclase signalling pathway in yeast: the relationship to nutrient-induced cell cycle control. Mol Microbiol 5, 1301-1307.

Thevelein, J. M. (1992). The RAS-adenylate cyclase pathway and cell cycle control in Saccharomyces cerevisiae. Antonie Leeunenboek 62, 109-130.
Thevelein, J. M. \& Beullens, M. (1985). Cyclic AMP and the stimulation of trehalase activity in the yeast Saccbaromyces cerevisiae by carbon sources, nitrogen sources and inhibitors of protein synthesis. J Gen Microbiol 131, 3199-3209.

Van Laere, A. (1989). Trehalose, reserve and/or stress metabolite? FEMS Microbiol Rev 63, 201-210.

Van Urk, H., Mak, P. R., Scheffers, W. A. \& Van Dijken, J. P. (1988). Metabolic responses of Saccharomyces cerevisiae CBS 8066 and Candida utilis CBS 621 upon transition from glucose limitation to glucose excess. Yeast 4, 283-291.

Wiemken, A. (1990). Trehalose in yeast, stress protectant rather than reserve carbohydrate. Antonie Leeuwenboek 58, 209-217.

Wills, C. (1990). Regulation of sugar and ethanol metabolism in Saccharomyces cerevisiae. Crit Rev Biochem Mol Biol 25, 245-280.

Received 19 July 1994; revised 14 October 1994; accepted 28 October 1994. 